\title{
THE ROTATIONAL EXCITATION OF OH IN ORION
}

\author{
E. F. van Dishoeck and G.J. Melnick \\ Center for Astrophysics, Cambridge MA 02138
}

J.H. Black

Steward Observatory, Tucson AZ 85721

The Orion-KL region has been studied extensively by observations of lines originating from highly-excited rotational levels of CO (see Watson et al. 1985). The lines arise in the shocked gas that lies at the interface of the hypersonic mass outflow of a newly-formed star and the quiescent material in Orion. The CO line intensities are sensitive to the physical conditions in the shocked region, and have been interpreted with a kinetic temperature $T \simeq 600 \mathrm{~K}$ and density $n\left(\mathrm{H}_{2}\right) \simeq 2 \times 10^{6}$ $\mathrm{cm}^{-3}$. Recently, pure rotational lines of $\mathrm{OH}$ have been detected as well in the farinfrared spectrum of Orion. Since the $\mathrm{OH}$ and $\mathrm{CO}$ molecules differ substantially in their energy level structure and in other properties, it is of interest to investigate whether the $\mathrm{OH}$ excitation probes the same physical conditions as the $\mathrm{CO}$ excitation or whether the two molecules reside in different parts of the shock.

Observations have been reported previously of the $\mathrm{OH}^{2} \Pi_{3 / 2} J=5 / 2 \rightarrow 3 / 2$ doublet at $119 \mu \mathrm{m}$ (Storey et al. 1981), the $J=7 / 2 \rightarrow 5 / 2$ doublet at $84 \mu \mathrm{m}$ (Watson et al. 1985, Viscuso et al. 1985a) and the ${ }^{2} \Pi_{1 / 2} J=3 / 2 \rightarrow 1 / 2$ doublet at $163 \mu \mathrm{m}$ (Viscuso et al. 1985b). Recent new measurements at high resolution $(\Delta \mathrm{v}=15 \mathrm{~km}$ $\mathrm{s}^{-1}$ ) have been made of the $163 \mu \mathrm{m}$ doublet, and these line profiles were compared with those of the adjacent $\mathrm{CO} J=16 \rightarrow 15$ line at $163 \mu \mathrm{m}$ and the $[\mathrm{OI}]^{3} \mathrm{P}_{1} \rightarrow{ }^{3} \mathrm{P}_{2}$ line at $63 \mu \mathrm{m}$. The $\mathrm{OH}$ lines are well resolved with intrinsic line widths of about $50 \mathrm{~km} \mathrm{~s}^{-1}$, somewhat larger than the $\mathrm{CO}$ and [OI] emission widths of about 30 $\mathrm{km} \mathrm{s}^{-1}$. In an effort to assess whether any significant fraction of the observed $\mathrm{OH}$ emission arises in a gas with $\mathrm{n}_{\mathrm{H}} \geq 10^{8} \mathrm{~cm}^{-3}$, searches have also been made for the higher excitation ${ }^{2} \Pi_{1 / 2} J=9 / 2 \rightarrow 7 / 2$ doublet at $55 \mu \mathrm{m}$ and the $J=11 / 2 \rightarrow 9 / 2$ doublet at $46 \mu \mathrm{m}$. The $55 \mu \mathrm{m}$ observations have yielded upper limits on the emitted flux in a 30" beam consistent with emission from gas with $\mathrm{n}_{\mathrm{H}}<10^{8} \mathrm{~cm}^{-3}$. The $46 \mu \mathrm{m}$ line strengths appear to be anomalously large $\left(\sim 10^{-17} \mathrm{~W} \mathrm{~cm}^{-2}\right)$ and may result from instrumental calibration problems. Attempts will be made to re-observe this doublet in the coming year. These observations are discussed in greater detail in Crawford et al. (1986), Melnick et al. (1986), and Melnick et al. (1986a).

Calculations of the steady-state populations have been performed for 36 levels of $\mathrm{OH}$ lying within $2000 \mathrm{~cm}^{-1}(\Delta \mathrm{E} / \mathrm{k} \leq 2900 \mathrm{~K})$ in excitation energy of the ground state, including the $\Lambda$-doubling, but neglecting the hyperfine interaction. The statistical equilibrium equations were solved taking into account the competing effects of absorption and stimulated emission in an ambient thermal dust radiation 
field, and of collisional excitation and deexcitation. Because of the large dipole moment of $\mathrm{OH}$, the radiative processes prove to be important in determining the level populations, in contrast with the $\mathrm{CO}$ excitation. The radiative transfer in the lines has been treated both within a uniform cloud, escape probability formulation, and in the Sobolev (large velocity gradient) approximation for a specific model of the Orion shock (cf. Draine and Roberge 1982). Results for the infrared line intensities in the 30-160 $\mu \mathrm{m}$ wavelength region have been presented for a range of densities, temperatures and $\mathrm{OH}$ column densities (see Black and van Dishoeck 1986).

The comparison with observations is complicated by the fact that the location of the continuum radiation with respect to the line forming region is not known, and by the possibility that the gas is highly clumped. If the continuum radiation is not directly behind the shock, the observed fluxes in the 119 and $84 \mu \mathrm{m}$ doublets in the Orion-KL region can be reproduced - within the factor two uncertainty in some of the collisional cross sections - with $n\left(\mathrm{H}_{2}\right) \simeq 3 \times 10^{6} \mathrm{~cm}^{-3}, T \simeq 750 \mathrm{~K}$ and $N(\mathrm{OH}) \simeq 10^{16} \mathrm{~cm}^{-2}$, in harmony with the interpretation of the CO lines. The intense flux in the $163 \mu \mathrm{m}$ doublet appears to require a somewhat larger density or $\mathrm{OH}$ column density. For $N(\mathrm{OH}) \simeq 10^{16} \mathrm{~cm}^{-2}$, the optical depth in the $119 \mu \mathrm{m}$ lines is about $10-20$, in the $84 \mu \mathrm{m}$ lines $1-2$ and in the $163 \mu \mathrm{m}$ lines $0.3-0.7$. The observed fluxes for all three doublets are also consistent, however, with a much lower temperature $T \simeq 75 \mathrm{~K}$ and a somewhat larger density $n\left(\mathrm{H}_{2}\right) \geq 10^{7} \mathrm{~cm}^{-3}$. If all of the infrared continuum radiation is located directly behind the shock, the observations are not consistent with the present models of the Orion shocked region based on the $\mathrm{CO}$ observations. Larger densities, $n\left(\mathrm{H}_{2}\right)>5 \times 10^{6} \mathrm{~cm}^{-3}$ and/or column densities, $N(\mathrm{OH})>10^{16} \mathrm{~cm}^{-2}$, are needed in this case to bring the 119 and $84 \mu \mathrm{m}$ lines into emission and to reproduce the observations. The models are consistent with the observed upper limit on the flux in the $55 \mu \mathrm{m}$ doublet, but they cannot reproduce the possibly large flux in the $46 \mu \mathrm{m}$ doublet. In all models, the lines involving the cross ladder transitions at 79 and $53 \mu \mathrm{m}$ are predicted to have comparable strengths to the 119 and $84 \mu \mathrm{m}$ lines. Further observational searches of lines involving these transitions, as well as higher $J$ transitions within one ladder, are needed to test the various models.

\section{REFERENCES}

Black, J.H. and van Dishoeck, E.F. 1986, to appear in "Masers, Molecules and Mass Outflows", Haystack Observatory conference 1985; in prep.

Crawford, M.K., Lugten, J.B., Fitelson, W., Genzel, R. and Melnick, G. 1986, preprint.

Draine, B.T. and Roberge, W.G. 1982, Ap. J. (Letters), 259, L91.

Melnick, G.J., Genzel, R., Crawford, M.K., and Lugten, J.B. 1986, in prep.

Melnick, G.J., Houck, J.R., Herter, T., Gull, G.E., and Graf, P. 1986a, in prep.

Storey, J.W.V., Watson, D.M. and Townes, C.H. 1981, Ap. J. (Letters), 244, L27.

Viscuso, P.J., Stacey, G.J., Harwit, M., Haas, M.R., Erickson, E.F. and Duffy, P.B. 1985 a, Ap. J., 296, 149.

Viscuso, P.J., Stacey, G.J., Fuller, C.E., Kurtz, N.T. and Harwit, M. 1985b, Ap. J., 296, 142.

Watson, D.M., Genzel, R., Townes, C.H. and Storey, J.W.V. 1985, preprint. 